\title{
BMJ Open Variation in access to community rehabilitation services and length of stay in hospital following a hip fracture: a cross-sectional study
}

\author{
Jenny Neuburger, ${ }^{1,2}$ Karen A Harding, ${ }^{3}$ Rachel J D Bradley, ${ }^{4}$ David A Cromwell, ${ }^{1,2}$ \\ Celia L Gregson ${ }^{5,6}$
}

To cite: Neuburger J, Harding KA, Bradley RJD, et al. Variation in access to community rehabilitation services and length of stay in hospital following a hip fracture: a cross-sectional study. BMJ Open 2014;4: e005469. doi:10.1136/ bmjopen-2014-005469

- Prepublication history and additional material is available. To view please visit the journal (http://dx.doi.org/ 10.1136/bmjopen-2014005469).

Received 14 April 2014 Revised 29 July 2014 Accepted 7 August 2014 CrossMark

For numbered affiliations see end of article.

Correspondence to Dr Jenny Neuburger; jenny.neuburger@Ishtm.ac.uk

\section{ABSTRACT}

Objectives: To assess variation in access to and use of community rehabilitation services for patients with a hip fracture, and whether this affects length of stay in hospital.

Design: Cross-sectional study using administrative patient-level data from Hospital Episode Statistics (HES) and organisational survey data.

Setting: A regional health economy in South West England served by four acute National Health Service (NHS) hospital trusts and six former Primary Care Trusts (PCTs).

Population: 1230 hip fracture patients treated in an acute hospital between 1 April 2011 and 29 February 2012.

Main outcomes: Information about access to community rehabilitation services for each acute hospital and PCT, reported by organisational survey.

Rates of patients transferred from acute hospital to community rehabilitation hospitals $(\mathrm{CRH})$ across eight groups with varying access; determined by acute hospital and PCT. Median lengths of stay in the acute hospital, and in the acute hospital plus $\mathrm{CRH}$ combined. Associations between the rate of transfer to a $\mathrm{CRH}$ and median lengths of stay assessed using Spearman's rank correlation coefficient $\left(r_{s}\right)$.

Results: Access to community rehabilitation services varied, including the number of $\mathrm{CRH}$ inpatient beds, formal access criteria and waiting times. In one PCT, no home-based rehabilitation service was available. The percentage of patients transferred to a $\mathrm{CRH}$ ranged from $2.1 \%$ to $54.7 \%$. A higher transfer rate was associated with a shorter median length of stay in the acute hospital $\left(r_{\mathrm{s}}=-0.8 ; p=0.01\right)$, but a longer median combined length of stay in the acute hospital and $\mathrm{CRH}$ $\left(r_{s}=+0.7 ; p=0.04\right)$.

Conclusions: Within one geographical area, there was wide variation in availability and use of community rehabilitation services for patients discharged from an acute hospital following a hip fracture. Reliance on transfers to community rehabilitation hospitals was associated with a longer length of stay in the NHS.

\section{Strengths and limitations of this study}

- This study is the first to combine data from acute hospitals and community rehabilitation hospitals (CRHs) to examine different institutional arrangements for providing rehabilitation care.

- The comprehensiveness of the Hospital Episode Statistics (HES) allowed us to match admissions of the same patient to acute and community rehabilitation beds.

- Our study relates to activity in one geographical area in South West England and may not be generalisable across the country.

- The HES database does not capture admissions to private hospitals. However, in England, almost all hip fractures are expected to pass through NHS care. Our organisational survey did not identify even occasional use of private residential rehabilitation services.

- Each of the acute hospitals had access to a small number of social care funded rehabilitation beds that are not captured within HES. This could lead to a slight underestimate of use of $\mathrm{CRH}$ beds and total institutional length of stay.

\section{INTRODUCTION}

An important element of ensuring a safe hospital discharge is the provision of appropriate services to support individuals in the community, including ongoing rehabilitation when indicated. ${ }^{1}$ When provided early, such services can reduce length of stay (LOS) in the acute hospital, although some frailer patients may benefit from extended inpatient rehabilitation to achieve a discharge home. However, the 2013 National Audit of Intermediate Care highlighted wide variation in the number, location and staffing of rehabilitation services across the country, ${ }^{2}$ with no improvement in capacity since the 
previous year. ${ }^{3}$ Little is known of the variation in access to community rehabilitation services for specific patient groups, and the impact on length of hospital stay.

Around 60000 older adults fracture a hip each year in England, ${ }^{4}$ and the number is projected to increase with our ageing population. Such fractures represent a major trauma for individuals and a significant societal burden, both through direct health service costs (UK estimated $£ 1.8$ billion in 2000), and important social sequelae. ${ }^{5}$ Since its launch in 2007, the National Hip Fracture Database (NHFD) supported by the 'Blue Book', has highlighted the importance of geriatrician-led multidisciplinary rehabilitation. ${ }^{1}$ In 2012, the National Institute for Health and Care Excellence (NICE) issued specific guidance (CMG46) on commissioning high-quality postacute hip fracture care for up to 6 weeks following hospital discharge. ${ }^{6}$

The 2013 NHFD report revealed wide variation in institutional arrangements for providing rehabilitation care following hip fracture, and wide variation in LOS following a hip fracture: the average LOS in NHS hospitals was 22 days, but this figure ranged from 12.9 to 33.5 days, nearly a threefold variation. ${ }^{4}$ With its focus on care provided within the acute hospital, the NHFD report was not able to identify external drivers of variation in length of stay, given the complex and heterogeneous provision of rehabilitation care. Ours is the first study to evaluate the impact of variation in community rehabilitation services, provided outside the acute hospital, on lengths of stay in the acute hospital and in the NHS overall.

We aimed to assess variation in access to and use of community rehabilitation services provided outside the acute hospital within one geographical area in England. First, we conducted an organisational survey to identify variation in access to community rehabilitation services across four NHS acute hospitals and their affiliated former Primary Care Trusts (PCTs). Second, we analysed administrative patient-level data from the Hospital Episode Statistics (HES) to calculate rates of transfer from acute hospitals to community rehabilitation hospitals across eight patient groups categorised by combinations of their acute hospital and their PCT. Third, we analysed the relationship between rate of transfer and average lengths of stay, in order to evaluate the efficiency of different institutional arrangements for providing rehabilitation care within the NHS.

\section{METHODS}

\section{Study setting}

Our study focused on one defined geographical area in South West England served by four NHS acute hospital trusts. One inner-city teaching hospital (hospital A) and three district general hospitals (hospitals B, C, D) served a combined total catchment population of approximately 1.7 million people. The acute hospitals represent four distinct models of inpatient hip fracture care with access to a range of community rehabilitation services provided by the acute trust or by former PCT. We defined a community rehabilitation hospital ( $\mathrm{CRH})$ as a local NHS institution providing on-site integrated health and social care with specifically inpatient access to physiotherapy for the purpose of rehabilitation; this contrasts with home-based rehabilitation and care services provided after discharge from a hospital in a patient's own home.

\section{Organisational survey of orthogeriatricians regarding access to community rehabilitation services}

A standardised questionnaire was used to facilitate structured interviews with hospital orthogeriatricians, all conducted by one orthogeriatrician (CLG), collecting retrospective data regarding provision of orthogeriatric and local community rehabilitation services in 2011/ 2012. Information was collected regarding: the orthogeriatric service model, source PCTs for admissions; CRHs and other residential intermediate care facilities to which patients could be transferred or discharged within each acute trust and PCT; availability of home-based community rehabilitation services within each PCT and access criteria; and ongoing clinical or managerial responsibility for patients following transfer to a CRH. Respondents were also given the opportunity to comment on other system issues relating to access (see online supplementary appendix). This survey information was combined with 2011 Census data on PCT catchment populations to crudely estimate numbers of community rehabilitation beds per 10000 people aged 65 years and over. ${ }^{7}$

\section{HES used in statistical analysis}

The flow of patients from acute hospitals to CRHs was established using an extract of patient-level data extracted from an anonymised copy of the HES database. The HES database contains administrative records describing the care of all hip fracture patients admitted to hospitals providing NHS-funded care in England, including acute and community hospitals. ${ }^{8}$ Patients' diagnoses are coded using ICD-10 (International Classification of Diseases, 10th revision), and procedures are coded using the classification of surgical operations from the UK Office of Population Censuses and Surveys (OPCS), V.4.

We identified patients who had a fractured neck of femur using the ICD-10 disease codes: S72.0 (fracture of neck of femur); S72.1 (pertrochanteric fracture); and S72.2 (subtrochanteric fracture). An anonymised patient identifier, derived from the patient's NHS number, was used to match admissions of the same patient to different hospitals.

Our sample included 1230 patients who met the following inclusion criteria: admitted to one of four acute trusts with a fractured neck of femur between 1 April 2011 and 29 February 2012 (11 months used to avoid downward bias of estimates of LOS due to truncation of 
Figure 1 Selection of patients from Hospital Episode Statistics.
1,615 patients treated for a hip fracture in one of four acute hospitals between $1^{\text {st }}$ April 2011 and $29^{\text {th }}$ February 2012

\section{Exclusions $(\mathbf{N}=\mathbf{3 8 5})$}

Registered to GP outside seven PCT areas comprising main catchment populations $(\mathrm{N}=107)$

Registered to GP in PCT outside main hospital catchment area (N $=29$ )

Registered to GP in PCT to which high rate of patients discharged to another NHS institution had a missing consecutive episode $(\mathrm{N}=$ 210)

Discharge destination from trust coded as another NHS institution, but missing consecutive episode $(\mathrm{N}=36)$

Missing Index of Multiple Deprivation $(\mathrm{N}=3)$

1,230 patients included in statistical analysis spells at 30 March 2012); and registered with a general practitioner (GP) in one of the seven PCT areas. A patient's PCT was defined by the address of their registered GP, because this determined formal eligibility for services. For patients who had their discharge destination coded as a transfer from the acute hospital to another NHS provider, we excluded those missing a record for a subsequent admission to a CRH or other NHS hospital. We excluded all patients from one PCT affiliated with hospital D because a third of CRH records were missing (figure 1).

\section{Variable definitions: CRH and lengths of stay}

We defined a transfer to a CRH bed as either: a formal discharge from the NHS acute trust and admission to a community hospital outside the trust; or a transfer within the same acute trust from the acute hospital to another site providing geriatric care, intermediate care or rehabilitation. In order to identify these transfers, we matched admissions of the same patient to different hospitals using the following criteria: the discharge destination code for the acute hospital or else the source/ method of admission code for the second hospital indicated a transfer; and the admission to the second hospital was not coded as an emergency admission.

We calculated LOS in the acute hospital as the number of days between the admission date to the acute hospital and the date of discharge from, or transfer out of, the acute hospital. We calculated the combined LOS in the acute hospital and CRH as the number of days between the date of admission to the acute hospital and the final date of discharge, from the CRH if the patient was transferred, or from the acute hospital if the patient was not transferred.
We derived other variables to describe patient characteristics, including: age (as a categorical variable: under 60; 60-69; 70-79; 80-89; 90 years and over); gender; comorbidity; socioeconomic deprivation; and living in a rural area. We used the Royal College of Surgeons of England's modified Charlson Score to calculate a comorbidity score. ${ }^{9}$ This is based on a number of selected chronic conditions identified using ICD-10 diagnosis codes in HES for the index admission and admissions during the previous year. We used the Index of Multiple Deprivation (IMD) to measure socioeconomic deprivation. ${ }^{10}$ We used the IMD score for a patient's area of residence and then grouped patients into five categories based on the national ranking of local areas. We used the classification of output areas as rural or urban, with a rural area defined as a village, hamlet or isolated dwelling.

\section{Statistical analysis}

We calculated the rate of transfer from the acute hospital to CRHs for each of eight patient groups categorised by combinations of their acute hospital and PCT. We categorised the data in this way because it is the combination of acute hospital and PCT that determines patient access to community rehabilitation services. For example, access will depend on formal and informal institutional arrangements such as: whether the acute hospital is part of a larger organisational unit (acute NHS trust) running its own CRH; agreed eligibility criteria and referral arrangements between providers; proximity of services to a patient's home; and waiting times for CRH beds.

We used the $\chi^{2}$ test to assess differences in transfer rates between groups. We used Spearman's rank correlation coefficient $r_{s}$ to measure associations between the transfer rate and median LOS in the acute hospital; and 
median LOS in the acute hospital and CRH combined, across the eight groups. We also checked correlations between adjusted measures (see online supplementary appendix for details). Data were analysed using Stata V.11. Reported $\mathrm{p}$ values are two sided.

\section{RESULTS}

\section{Acute hospital services}

Each hospital admitted between 300 and 400 patients with hip fracture each year. The three district general hospitals provided a model of joint care between orthogeriatric and trauma and orthopaedic services, while the teaching hospital provided a liaison model of orthogeriatric care. Timetabled orthogeriatrician input varied, with the fewest clinical sessions being provided within the teaching hospital and between 7 and 13 sessions provided within the district general hospitals (table 1).

\section{Access to community rehabilitation services}

The six PCTs served a population of around half a million older adults (aged $\geq 65$ years). Each PCT was served by one or two acute hospitals and had access to between one and three CRHs. The total number of CRH beds per PCT ranged from 18 to 82, and the number of CRH beds available per 10000 older adults ranged from 4 to 20 (see online data supplement, table A). Each hospital was located in PCTs served by between two and nine CRHs (table 1).

Access to CRH beds depended on the allocation of beds and referral arrangements, not only on the number of beds. One of the acute hospitals (hospital A) was part of a larger acute trust that ran its own CRH. In one PCT (PCT 1) only patients registered with specific GPs in one town had access to the PCT-run CRH. The two CRHs affiliated with hospital B had very limited availability and long waiting times, with the result that most patients were fit to return home before a CRH bed became available.

All PCTs except for one (PCT 6) offered home-based community rehabilitation services. One PCT (PCT 3) had an early supported discharge programme, but this operated strict access criteria including: no cognitive deficit; safe to mobilise with aids; and no over-night care needs.

No acute hospital orthogeriatician retained clinical or managerial responsibilities for ongoing rehabilitation of patients following their transfer to a CRH.

\section{Characteristics of patients sustaining a hip fracture}

Out of 1230 patients included in the sample, just under a third were male, approximately a fifth were aged 90 years or older, and more than half had at least one comorbid condition included in the Charlson score (table 2). Rural living and socioeconomic deprivation varied between acute hospital hip fracture populations. Two of the acute hospitals treated a mix of patients who lived in deprived and affluent areas, but where only $4 \%$ lived in rural areas. In contrast, the other two acute hospitals served more affluent populations, with $15.5 \%$ and $23.7 \%$ from rural areas, respectively.

\section{Variation in rates of transfer to $\mathrm{CRH}$ beds}

Table 3 displays the transfer rates to CRH beds across eight patient groups according to combinations of their acute hospital and PCT. Rates of transfer ranged from $2.1 \%$ to $54.7 \%$, representing large and statistically significant variation $(\mathrm{p}<0.001)$.

Comparing these figures to the organisational survey findings, the patient groups with the lowest transfer rates were treated in an acute hospital (hospital B) that reported very poor access to CRH beds for its patients across both its PCTs. The patient group with the highest CRH transfer rate (hospital D, transferring to PCT 6) occurred in the only PCT without access to a homebased rehabilitation service.

\section{Influence of transfer rate to CRH on LOS}

Median LOS in the acute hospital ranged from 11 to 19 days. Median combined LOS in the acute hospital and CRH ranged from 17 to 27.5 days. A higher rate of CRH transfer was associated with a shorter median LOS

Table 1 Description of four acute hospital hip fracture services and access to community rehabilitation services, information collected via organisational survey $2011 / 2012$

\begin{tabular}{|c|c|c|c|c|}
\hline & Hospital A & Hospital B & Hospital C & Hospital D \\
\hline Approximate catchment population & 300000 & 500000 & 400000 & 500000 \\
\hline Teaching/DGH & Teaching & $\mathrm{DGH}$ & $\mathrm{DGH}$ & $\mathrm{DGH}$ \\
\hline Orthogeriatrician input & Liaison & Joint care & Joint care & Joint care \\
\hline Orthogeriatrican sessions*/week & 3 & 7 & 13 & $10 \dagger$ \\
\hline Number of PCTs at discharge $\ddagger$ & 3 & 3 & 2 & 4 \\
\hline Number of PCTs with a home-based rehabilitation service & 3 & 3 & 2 & 3 \\
\hline Number of $\mathrm{CRH}$ s at discharge $\ddagger, \S$ & 3 & 2 & 7 & 9 \\
\hline \multicolumn{5}{|c|}{$\begin{array}{l}\text { *One session=four hours of either morning or afternoon work. } \\
\text { tIncludes five sessions provided by a specialist trainee. } \\
\text { †Hospitals may discharge small numbers of patients to other PCTs. } \\
\text { §Each hospital also had access to a small number of beds in one additional social care funded community rehabilitation facility. } \\
\text { CRH, community rehabilitation hospital; DGH, district general hospital; Joint care, formal joint care between orthogeriatric and trauma and } \\
\text { orthopaedic services; PCT, Primary Care Trusts. }\end{array}$} \\
\hline
\end{tabular}


Table 2 Description of characteristics of hip fracture patients treated in four acute hospitals included in analysis, Hospital Episode Statistics (HES) data 1 April 2011 to 29 February 2012

\begin{tabular}{|c|c|c|c|c|}
\hline & Hospital A & Hospital B & Hospital C & Hospital D \\
\hline Number of patients & 303 & 326 & 348 & 253 \\
\hline Female, $\mathrm{n}(\%)$ & $220(72.6)$ & $249(76.4)$ & $246(70.7)$ & $191(75.5)$ \\
\hline \multicolumn{5}{|l|}{ Age in years, $n(\%)$} \\
\hline $50-59$ & $9(3.0)$ & $10(3.1)$ & $13(3.7)$ & $8(3.2)$ \\
\hline $60-69$ & $28(9.2)$ & $22(6.8)$ & $28(8.1)$ & $22(8.7)$ \\
\hline $70-79$ & $58(19.1)$ & $63(19.3)$ & $72(20.7)$ & $48(19.0)$ \\
\hline $80-89$ & $152(50.2)$ & $168(51.5)$ & $165(47.4)$ & $122(48.2)$ \\
\hline 90 and older & $56(18.4)$ & 63 (19.3) & 70 (20.1) & $53(21.0)$ \\
\hline \multicolumn{5}{|c|}{ Number of comorbidities, $\mathrm{n}(\%)^{*}$} \\
\hline 0 & $135(44.6)$ & $156(47.9)$ & $148(42.5)$ & $99(39.1)$ \\
\hline 1 & $117(38.6)$ & $121(37.1)$ & $142(40.8)$ & $106(41.9)$ \\
\hline$\geq 2$ & $51(16.8)$ & $49(15.0)$ & $58(16.7)$ & $48(19.0)$ \\
\hline \multicolumn{5}{|c|}{ Index of multiple deprivation, $\mathrm{n}(\%)$} \\
\hline 1 (least deprived) & $62(20.5)$ & $91(27.9)$ & $121(34.8)$ & $61(24.0)$ \\
\hline 2 & $67(22.1)$ & 55 (16.9) & $101(29.0)$ & $82(32.4)$ \\
\hline 3 & 39 (12.9) & $61(18.7)$ & 60 (17.2) & $61(24.1)$ \\
\hline 4 & $76(25.1)$ & $81(24.9)$ & 38 (10.9) & $44(17.4)$ \\
\hline 5 (most deprived) & 59 (19.5) & $38(11.6)$ & $28(8.0)$ & $5(2.0)$ \\
\hline Living in rural area, $\mathrm{n}(\%)$ & $11(3.6)$ & $15(4.6)$ & $54(15.5)$ & $60(23.7)$ \\
\hline
\end{tabular}

in the acute hospital $\left(\mathrm{r}_{\mathrm{s}}=-0.8 ; \mathrm{p}=0.01\right)$, but a longer combined LOS $\left(\mathrm{r}_{\mathrm{s}}=+0.7 ; \mathrm{p}=0.04\right)$. While there was a clear linear relationship between higher CRH transfer rates and reduced acute hospital LOS, the relationship with combined length of stay did not appear to be linear. Only high transfer rates seemed to be associated with a more marked increase in combined LOS (figure 2).

Adjustment for age, gender, comorbidity, socioeconomic deprivation and rural habitation did not change the associations between transfer rates and LOS (see online supplementary appendix).

\section{DISCUSSION}

\section{Main findings of this study}

In a geographical area of England that covered half a million older adults, we identified considerable variation in access to and use of community rehabilitation services following acute hospitalisation for a hip fracture. Across the PCTs, the number of CRH beds available ranged from 4 to 20 beds per 10000 older adults (aged $\geq 65$ years). Access criteria also varied; for example, in one PCT, only patients registered with specific GPs in one town had access to the CRH beds. Rates of transfer from the acute hospital to a CRH ranged from $2.1 \%$ to $54.7 \%$ between patient groups with different levels of access to community rehabilitation services.

Variation in transfer rates to $\mathrm{CRH}$ beds was in turn associated with differences in median hospital length of stay. A higher rate of CRH transfer was associated with a shorter average LOS in the acute hospital, but a longer average combined LOS in the acute hospital and CRH.

Table 3 Relationship between rate of transfer to CRH and LOS, across eight groups categorised by the combination of acute hospital and PCT

\begin{tabular}{lllcll}
\hline Group* $^{*}$ & $\begin{array}{l}\text { Acute hospital } \\
\text { and PCT }\end{array}$ & $\begin{array}{l}\text { Number of } \\
\text { patients }\end{array}$ & $\begin{array}{l}\text { Number (\%) of patients } \\
\text { transferred to CRH }\end{array}$ & $\begin{array}{l}\text { Median acute } \\
\text { hospital LOS (days) }\end{array}$ & $\begin{array}{l}\text { Median combined } \\
\text { LOS (days)† }\end{array}$ \\
\hline 1 & Hospital B, PCT 2 & 141 & $3(2.1)$ & 19 & 20 \\
2 & Hospital B, PCT 3 & 185 & $8(4.3)$ & 19 & 19 \\
3 & Hospital C, PCT 5 & 137 & $44(32.1)$ & 13 & 17 \\
4 & Hospital A, PCT 1† & 70 & $23(32.9)$ & 13 & 25.5 \\
5 & Hospital C, PCT 4 & 211 & $80(37.9)$ & 13 & 23 \\
6 & Hospital D, PCT 5 & 189 & $73(38.6)$ & 14 & 21 \\
7 & Hospital A, PCT 2† & 233 & $110(47.2)$ & 11 & 23 \\
8 & Hospital D, PCT 6 & 64 & $35(54.7)$ & 11 & 27.5 \\
\hline
\end{tabular}

*Group ranked by rate of transfer to the $\mathrm{CRH}$.

†This is the median combined length of stay in the acute hospital and the $\mathrm{CRH}$.

$\ddagger$ These represent transfers from the acute hospital to a CRH run by the acute hospital trust.

$\mathrm{CRH}$, community rehabilitation hospitals; LOS, length of stay; PCT, Primary Care Trust. 

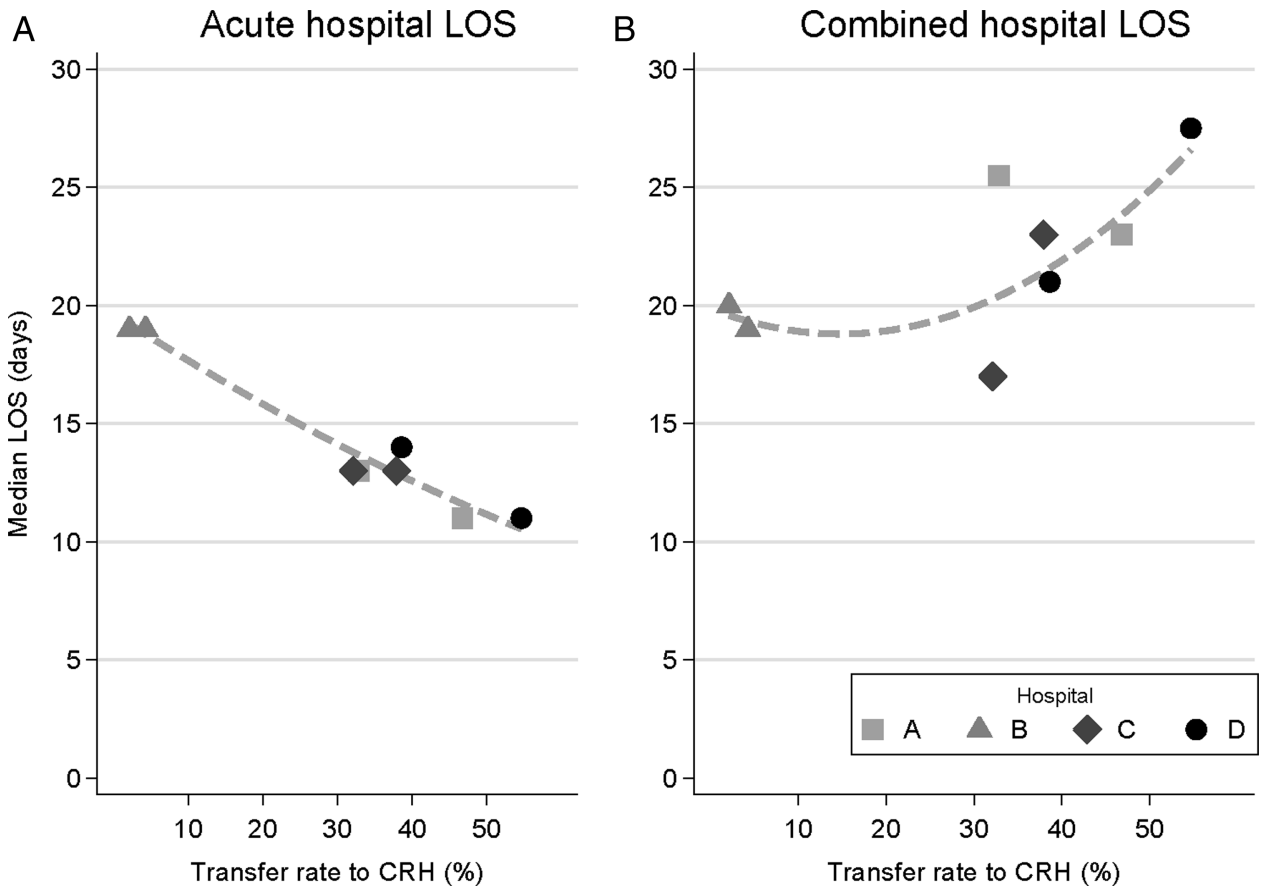

Figure 2 Relationship between rate of transfer to community rehabilitation hospital (CRH) and median length of stay (LOS) in: (A) acute hospital; and $(\mathrm{B})$ acute hospital and $\mathrm{CRH}$ combined. Each of the eight points on each graph shows the transfer rate to a community rehabilitation hospital $(\mathrm{CRH})$ and median length of stay (LOS) for patient groups categorised by combinations of their acute hospital and Primary Care Trust (also see table 3). The dashed lines are quadratic fits of LOS to transfer rate, included for illustrative purposes.

\section{Findings in context}

From a patient's perspective, the advantages of transfer to a CRH may include being closer to home, family and friends, as well as local community rehabilitation teams; factors potentially of greater importance in geographically remote rural areas. ${ }^{11}$ This may explain why the two hospitals $(\mathrm{C}$ and $\mathrm{D})$ with larger rural populations transferred more patients to CRHs. Balanced against this are possible negative aspects of transfer, such as: delayed rehabilitation progress due to the disruption of a transfer; the need to become familiar with another healthcare team; and the risk of less intensive rehabilitation in slower-stream units.

In England and Wales, NICE has issued specific guidance regarding commissioning of high-quality hip fracture care for up to 6 weeks following hospital discharge, stating patients should be offered early supported discharge when appropriate ${ }^{6}$; a service we found to be available in only one PCT. NICE also state that continued rehabilitation in a CRH should only be considered if the hip fracture clinical team retains managerial responsibility, ensuring that CRHs are not used as a substitute for effective acute hospital rehabilitation. However, in our study, no hospital orthogeriatrician retained clinical or managerial responsibility for ongoing rehabilitation following transfer from the acute hospital. As transfers often involve moves between organisations and hence lines of accountability and employment, this is perhaps not surprising. The increasing development of community geriatricians may offer some scope to improve continuity of care. ${ }^{12}$

\section{What is already known on this topic?}

There is some evidence from the UK on the relative efficiency and effectiveness of different institutional arrangements for providing postfracture rehabilitation care. A study of eight hospitals in East Anglia found patients treated in hospitals with a policy of transferring to other wards prior to discharge had a longer average LOS. ${ }^{13}$ A comparison of two hospitals found that routine transfer of patients to a Geriatric Orthopaedic Rehabilitation Unit in one hospital was associated with a shorter average stay on the orthopaedic ward but a longer hospital stay. ${ }^{14}$ The introduction of the Peterborough hip fracture service in 1986, including the 'Hospital at Home' scheme, increased the proportion of patients discharged directly home over an 11-year period from $50 \%$ to $86 \%$, reduced transfer rates to other wards from $43 \%$ to $9 \%$, and decreased LOS from 51 to 21 days. $^{15}$

In the US, the numbers of rehabilitation facilities and distance from a patient's home have been identified as stronger determinants of where patients received postacute rehabilitation than individual characteristics. ${ }^{16}{ }^{17} \mathrm{~A}$ systematic review of 30 randomised and 25 nonrandomised studies of hip fracture rehabilitation concluded that clinical pathways providing intensive therapy and early supported discharge were associated with 
improved functional recovery, while less intense postacute 'skilled nursing facility' rehabilitation was associated with a longer combined LOS. ${ }^{18}$

\section{Study strengths and limitations}

This study is the first to combine data from acute and community rehabilitation hospitals to examine the effect of different institutional arrangements for providing rehabilitation care within the NHS. Its strengths come from using interviews to establish a detailed picture of service provision and from using administrative patientlevel data to describe the flow of patients between acute and rehabilitation hospitals. The comprehensiveness of HES allowed us to match admissions of the same patient to acute and CRH beds within the NHS.

We excluded patients discharged from hospital D to one PCT, since nearly a third, coded as transfers to another NHS institution, were missing a subsequent CRH admission record. We re-ran the analysis using more complete data from the previous year, including this PCT, which did not alter conclusions about the relationship between CRH transfers and LOS.

HES do not capture admissions to private hospitals. However, in England, almost all hip fractures are expected to pass through NHS care. Our organisational survey did not identify even occasional use of private residential rehabilitation services. Each of the acute hospitals did have access to a small number of social care funded rehabilitation beds not captured within HES, which could lead to a slight underestimate of CRH bed usage.

We have presented results from simple analyses of the crude rates of transfer and median LOS across eight patient groups with varying levels of access to community rehabilitation services. We also calculated variation in adjusted transfer rates and correlations between these and adjusted LOS, taking account of age, sex, comorbidity, socioeconomic deprivation and rural living, but this did not alter our conclusions (see online supplementary appendix). However, HES lacks data on some potential confounders such as prefracture mobility, and the need for new nursing home placement. Based on NHFD published data, across the four acute hospitals the proportion of patients admitted from a nursing home or residential care was similar (17-19\%), but the proportion discharged from the acute trust to a nursing home or residential care was more variable $(14-24 \%) .{ }^{19}$ To what extent delays waiting for new nursing home placements impact on median LOS in unclear, although use of median rather than mean, reduces the influence of outliers. We included the small proportion of patients who were managed without surgery and who died in hospital; but their inclusion or exclusion did not affect our study conclusions.

Finally, our study relates to activity in one geographical area in South West England, and to former PCTs now replaced by Clinical Commissioning Groups (CCGs), and may not be generalisable across the country. On the other hand, the study population had similar demographic characteristics to the national hip fracture population and contained similar between-hospital variation in acute and combined lengths of stay ${ }^{4}$; furthermore, the new CCGs in this region mirror the old PCTs, hence we judged our analysis to still be relevant to the 'New NHS'.

\section{Unanswered questions for future research}

Our study focused on one English region. The results provide insight into the relationship between availability and use of community rehabilitation services, and hospital LOS, but the generalisability of the results is limited. It is important that studies are undertaken in other locations to extend our understanding of how different sets of institutional arrangements for providing rehabilitation care affect LOS, as well as other outcomes. The extent to which financially-pressured hospital management systems drive CRH transfers over the influence of the clinical team is also not known. Given recent NHS reorganisation and move to clinical commissioning of services, it will be interesting to see how our findings change over the next few years.

\section{Conclusions and policy implications}

Access to post-fracture rehabilitation care varied within a geographical area in South West England. Inequity in access to community rehabilitation services is inconsistent with the government's strongly promoted policy of patient choice, aimed at reducing inequalities in access to healthcare, improving responsiveness and quality of services $^{20}$ and reducing the 'postcode lottery' in service provision for older people. $^{21}$

Poor access to home-based rehabilitation services is arguably inefficient, as well as inequitable. The highest rate of transfer to community rehabilitation hospitals was observed in one patient group without any access to a home-based community rehabilitation service. In turn, a high rate of transfer to community rehabilitation hospitals was associated with a shorter stay in the acute hospital, but at the expense of a longer combined LOS.

These findings have relevance to current clinical commissioning groups planning intermediate care services. In 2011/2012, the average cost of an excess acute hospital bed day was $£ 264,{ }^{22}$ with a CRH bed $£ 252 /$ day. $^{23}$ To illustrate, our estimates suggest that reducing CRH transfer rates from $50 \%$ to $20 \%$ for 20000 hip fracture patients could save the English NHS around $£ 19$ million per year (see online supplementary appendix for details). Balanced against this potential saving would be the costs of home-based rehabilitation care, often required for early hospital discharge, although homebased services have lower costs per service user. ${ }^{2}$

Appropriate and equitable commissioning of postfracture rehabilitation services is required, in collaboration with clinical teams, to ensure fair access that is governed by clinical need and patient choice rather than geography. Providing a range of rehabilitation options could also improve the efficiency of care. 
Author affiliations

${ }^{1}$ Department of Health Services Research \& Policy, London School of Hygiene \& Tropical Medicine, London, UK

${ }^{2}$ Clinical Effectiveness Unit, The Royal College of Surgeons of England, London, UK

${ }^{3}$ Frenchay Hospital, North Bristol NHS Trust, Bristol, UK

${ }^{4}$ University Hospitals Bristol NHS Foundation Trust, Bristol, UK

${ }^{5}$ Musculoskeletal Research Unit, School of Clinical Sciences, University of Bristol, Bristol, UK

${ }^{6}$ Older Person's Unit, Royal United Hospital Bath NHS Trust, Bath, UK

Acknowledgements Hospital episode statistics were made available by the NHS Health and Social Care Information Centre (Copyright 2012, Re-used with the permission of The Health and Social Care Information Centre. All rights reserved.) The authors thank Lynn Copley for providing the required extract from HES database.

Contributors JN and CLG conceived and designed the study and wrote the manuscript. CLG carried out the organisational survey. KAH, RJDB provided organisational data. JN performed the statistical analyses. JN, KAH, RJDB, DAC and CLG all commented on, revised sequential drafts and approved the final manuscript for publication. JN is guarantor

Funding National Institute for Health Research; Arthritis Research UK. JN is funded by a NIHR Post Doctoral Fellowship (PDF-2013-06-078); CLG is funded by Arthritis Research UK through a Clinician Scientist Fellowship (grant ref 20000).

Competing interests None.

Ethics approval The study is exempt from UK National Research Ethics Committee approval as it involved analysis of an existing dataset of anonymised data for service evaluation. Approvals for the use of anonymised Hospital Episode Statistics (HES) data were obtained as part of the standard approval process.

Provenance and peer review Not commissioned; externally peer reviewed.

Data sharing statement No additional data are available.

Open Access This is an Open Access article distributed in accordance with the Creative Commons Attribution Non Commercial (CC BY-NC 4.0) license, which permits others to distribute, remix, adapt, build upon this work noncommercially, and license their derivative works on different terms, provided the original work is properly cited and the use is non-commercial. See: http:// creativecommons.org/licenses/by-nc/4.0/

\section{REFERENCES}

1. British Orthopaedic Association, British Geriatric Society. The care of patients with fragility fracture. British Orthopaedic Association, 2007.

2. NHS Benchmarking Network. National Audit of Intermediate Care (NAIC) Report 2013. http://www.nhsbenchmarking.nhs.uk/
projects/partnership-projects/National-Audit-of-Intermediate-Care/ year-two.php

3. NHS Benchmarking Network. National Audit of Intermediate Care (NAIC) Report 2012. 2012.

4. Johansen AW, Wakeman R, Boulton C, et al. The National Hip Fracture Database National Report 2013. 2013. http://www.nhfd.co.uk/

5. Burge RT, Worley D, Johansen A, et al. The cost of osteoporotic fractures in the UK: projections for 2000-2020. J Med Econ 2001;4:51-62.

6. National Institute for Health and Clinical Excellence. CMG46: Management of hip fracture in adults. NICE, 2012.

7. Office for National Statistics. ONS Mid-2011 Population Estimates for Primary Care Organisations in England by Single Year of Age and Sex; based on the results of the 2011 Census. http://www.ons.gov. uk/ons/publications/re-reference-tables.html?edition=tcm\%3A77297507

8. Health and Social Care Information Centre. Hospital Episode Statistics. http://www.hscic.gov.uk/hes

9. Armitage JN, Van der Meulen JH; Royal College of Surgeons Co-morbidity Consensus Group. Identifying co-morbidity in surgical patients using administrative data with the Royal College of Surgeons Charlson Score. Br J Surg 2010;97:772-81.

10. Noble M, McLellan D, Wilkinson K, et al. The English indices of deprivation 2007. In: Government. DfCaL, editor.: HMSO, 2008

11. Dixon S, Nancarrow S, Enderby P, et al. Assessing patient preferences for the delivery of different community-based models of care using a discrete choice experiment. Health Expectations, 2013.

12. Eckford D. Foundation training in community geriatrics. BMJ Careers 2014. http://careers.bmj.com/careers/advice/view-article.html? $\mathrm{id}=20018142$

13. Parker MJ, Todd CJ, Palmer CR, et al. Inter-hospital variations in length of hospital stay following hip fracture. Age Ageing 1998;27:333-7.

14. Parker MJ, Lewis SJ, Mountain J, et al. Hip fracture rehabilitation-a comparison of two centres. Injury 2002;33:7-11.

15. Parker MJ, Pryor GA, Myles J. 11-year results in 2,846 patients of the Peterborough Hip Fracture Project. Acta Orthop Scand 2000;71:34-8.

16. Freburger JK, Holmes GM, Ku L-JE. Postacute rehabilitation care for hip fracture: who gets the most care? J Am Geriatr Soc 2012;60:1929-35.

17. Buntin MB, Garten AD, Paddock $S$, et al. How much is postacute care use affected by its availability? Health Serv Res 2005;40:413-34.

18. Chudyk AM, Jutai JW, Petrella RJ, et al. Systematic review of hip fracture rehabilitation practices in the elderly. Arch Phys Med Rehabi 2009;90:246-62.

19. Currie C M, Plant F, Roberts J, et al. The National Hip Fracture Database National Report 2012. 2012.

20. Dixon A, Robertson R, Appleby J, et al. Patient choice. The King's Fund, 2010

21. Currie CT. Health and social care of older people: could policy generalise good practice? J Integr Care 2010;18:20-7.

22. Department of Health. Falls and fractures: effective interventions in health and social care (best practice guidance). London: Department of Health, 2009.

23. Department of Health. Reference costs guidance for 2011-12. Department of Health, 2012. 